\title{
On the Enhanced Reverse Beta Processes in Graphene-Iron Composite Nanostructures at High Temperatures in Strong Magnetic Field
}

\author{
Reginald B. Little*
}

National High Magnetic Field Laboratory, Florida State University, Tallahassee, Florida, USA

\begin{abstract}
Strong dense many-spin interactions have been proposed to organize novel orbital dynamics (the Little Effect) for novel chemical and catalytic phenomena. The recent determinations of the relativistic and quantum Hall effects of carriers in graphene under strong magnetic confinement have substantiated the Little Effect. Moreover such nonclassical phenomena under the stronger magnetic confinement of ferro-nanocatalysts are here shown to organize reverse beta processes and possibly pycnonuclear reactions under high temperature and high-pressure conditions. Such processes have implications for reverse beta reactions and nuclear reactions within the earth's interior and new technologies for carbon nanotube-ferrometal and nanographene-ferrometal composites.
\end{abstract}

\section{INTRODUCTION}

In 2000, a physical basis for ferromagnetic carbon was determined on the basis of carbon orbital alterations (defects, disorder, distortion, strain and pentagon-heptagon ring defects) during carbon nanotube (CNT) nucleation and growth by ferro-nanocatalysts [1]. The ferromagnetism of the forming graphene and CNT was shown to assist the CNT coupling to the ferrocatalyst for the concerted organization of many carbon magnetic atomic orbitals into magnetic molecular graphitic orbitals by polarized spin currents and spin waves (the Little Effect) and their consequent dynamical electromagnetic induction between the ferro-catalyst and the nucleating and growing CNT. These dynamical magnetic and electric fields of the nanocatalyst due to quantized spin currents and spin waves were determined to effect quantum Hall type interactions (even at the synthesis temperature) between the forming graphene and the underlying ferrocatalyst that provide the needed quanta of energy and momenta to accelerate and organize the ring currents in the forming graphene and CNT. In support of this, other investigators have recently, experimentally and computationally demonstrated quantum Hall type effects in graphene even at room temperature $[2,3]$ with level spacing extrapolated for quantum effects to temperatures as high as $2800 \mathrm{~K}[3,4]$. In addition to the quantum dynamics, many investigators have also recently, experimentally and computationally demonstrated the relativistic nature of quasiparticles in graphene for the Dirac spin character of the electrons and holes [4-7]. The electron velocities in graphene have been determined to exceed $10^{-2} \mathrm{c}$ (1\% the speed of light) [5-7]. Here it is demonstrated that the energies of forming graphene are even greater during the bond rearrangements with associated dangling bonds, surface energy and surface tension due to the energetic accumulation from many broken bonds ( $>100,000$ fractured chemical bonds) by antisymmetry and Dirac spin confinement by correlation and exchange with an underlying

*Address correspondence to this author at the National High Magnetic Field Laboratory, Florida State University, Tallahassee, Florida, USA;

E-mail: redge_little@yahoo.com strong magnetic iron lattice. Weaker perpendicular, external man-made magnetic fields (20-45 Tesla) have been shown to effectively confine the Dirac relativistic spins in graphene $[8,9]$. But, here much stronger, transient Amperean ferromagnetic (thousands of Tesla) confinement by exchange and correlation with the underlying ferrometals are suggested to allow even tighter confinement and stronger dynamical quantum Hall effect of much higher energy Dirac spins during the ferro-catalytic graphene formation. The huge quantum Hall effects under the much stronger magnetic field of the iron lattice even cause resistance to electronic orbital motion (conduction at the molecular and atomic levels) to create heavier electrons of molecular and lattice orbital motions but high-speed, relativistic, revolutionary electronic intra-orbital motion with its spin (spinrevorbital motion). Such huge magnetic field of iron can even shift and mix the electronic orbital, revolutionary and spin motions (spinrevorbital) inside the Bohr radius for reverse beta to form neutrons. These recent findings provide strong support of the strong, dense spin-induced orbital dynamics known as the Little Effect and its account of CNT, diamond and reverse beta processes [10].

In this paper, results are presented from experiments on magnetized, hot, hydrogenated graphene-iron composites that test for the occurrence and enhancement of such reverse beta processes in the composite. This previously proposed model [10] for reverse beta in metal hydride lattices is again tested using a similar system that has been used to show diamond nucleation and growth (also an internal terrestrial process) from catalyzed carbon black at atmospheric pressure and high temperatures $\left(920{ }^{\circ} \mathrm{C}\right)$ with $\mathrm{Fe}$ catalyst and a hydrogenous atmosphere in strong static magnetic field of 17 Tesla [11].

\section{PROCEDURE}

A mixture of iron and carbon black was placed inside a sealed quartz vessel and installed within the bore of a DC magnet. The vessel was purged with flowing argon (Ar) and heated to $920{ }^{\circ} \mathrm{C}$. Hydrogen was then flowed over the $\mathrm{Fe}$ and carbon black and the Ar flow was terminated. After 10 min- 
utes of reduction of the catalyst by flowing hydrogen, the DC magnet was slowly ramped up to 17 Tesla. $\mathrm{CH}_{4}$ was also introduced with the $\mathrm{H}_{2}$ flow over the $\mathrm{Fe}$ and carbon black. The system was maintained at $920{ }^{\circ} \mathrm{C}$ and 17 Tesla in the flowing $\mathrm{H}_{2}$ and $\mathrm{CH}_{4}$ for 2.5 hours. Afterward, the Ar flow was restarted and the $\mathrm{CH}_{4}$ and $\mathrm{H}_{2}$ flows were terminated. The magnetic field was then ramped down to 0 tesla. The sample was then immediately removed from the magnet and cooled to room temperature by applying water to the outside of the quartz tube. The $\mathrm{H}, \mathrm{C}, \mathrm{Fe}$ samples were analyzed by secondary ion mass spectroscopy (SIMS). It is important to note that here strong magnetization of hydrogenated, carbonaceous iron at high temperatures is introduced as a system to mimic some of the internal conditions in some interior parts of the earth, although with slower kinetics due to the lower pressure.

\section{RESULTS AND DISCUSSIONS}

The high temperature $\left(920{ }^{\circ} \mathrm{C}\right)$ annealing of the $\mathrm{C}, \mathrm{Fe}$ sample under the strong magnetic field and a methanehydrogenous atmosphere causes catalytic chemical transformation of the carbon as the $\mathrm{Fe}$ dissolves the carbon and reprecipitates it as some graphite and some diamond [11]. The magnetization also allows the transient accumulation of high chemical and electronic energy (coulombic and magnetic) by antisymmetry of broken chemical bonds in the defective distorted graphene on the iron catalyst for substantial tremendous electroweak and electromagnetic interactions between the high-energy relativistic electrons and absorbed protons in the graphene-iron composite for reverse beta processes and neutron formation. The neutrons are absorbed by the surrounding hydrogen and carbon with the formations of ${ }^{2} \mathrm{D},{ }^{3} \mathrm{~T}$, and ${ }^{13} \mathrm{C}$. SIMS analysis of samples relative to a control determined the presence of these nuclides in anomalous relative amounts.

A typical SIMS of the H, C, Fe matrix before (control) the high temperature magnetization in hydrogenous atmosphere is shown in Fig. (1). The SIMS of the H, C, Fe matrix after (sample) the high temperature, magnetic annealing in hydrogenous atmosphere is shown in Fig. (2). Various differences develop in the mass spectrum of the sample after its magnetic, high temperature treatment in hydrogenous atmosphere. In general, distinct isotopic differences exist between the sample and control for hydrogen and carbon, which are the major components of the samples. Furthermore, the mass intensities (for many nuclides lighter than iron) are greater after the high temperature magnetization. On the one hand, the mass intensities for some nuclides

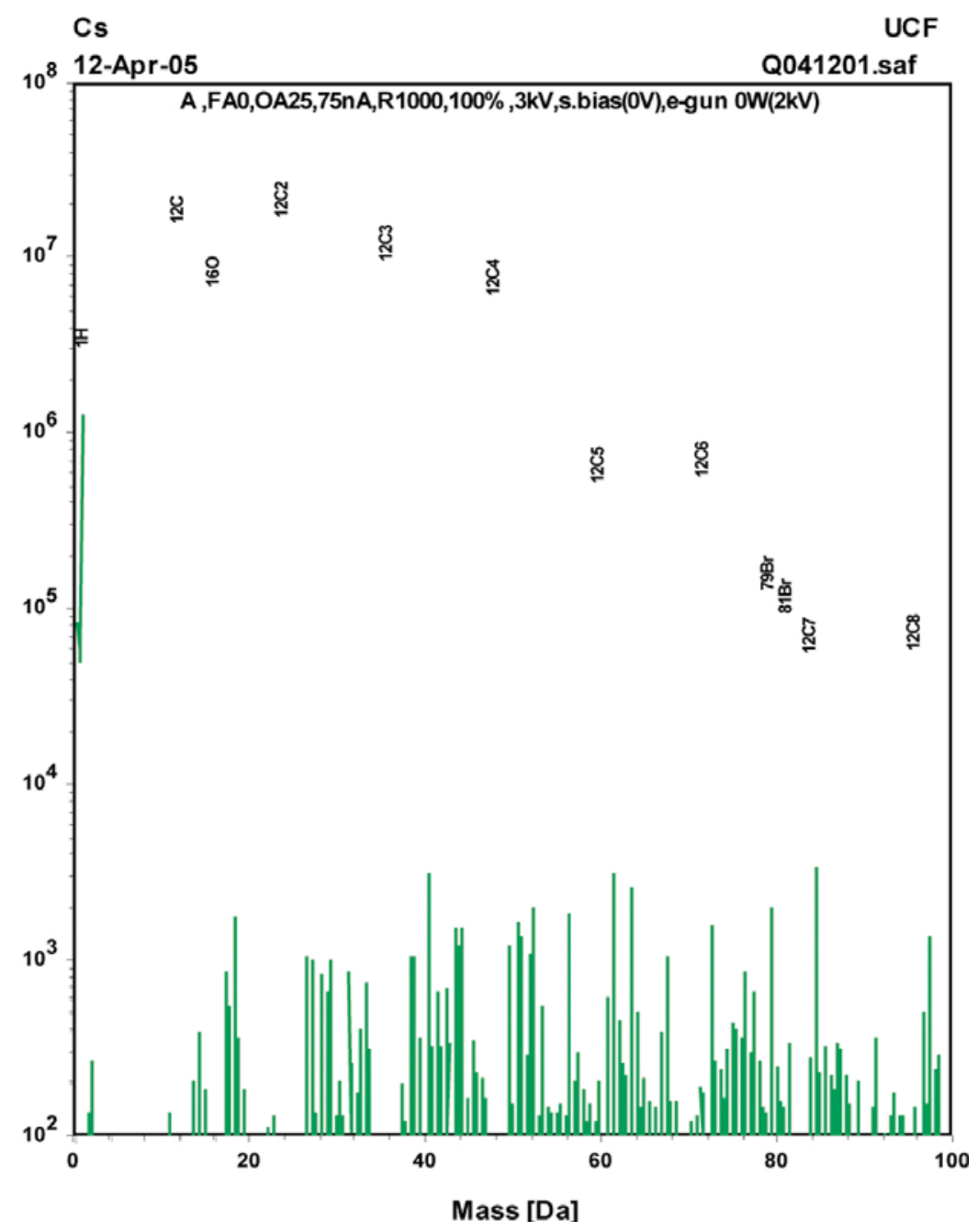

Fig. (1). SIMS of Hydrogen-Carbon-Iron Before High Temperature Magnetization (17 Tesla). 


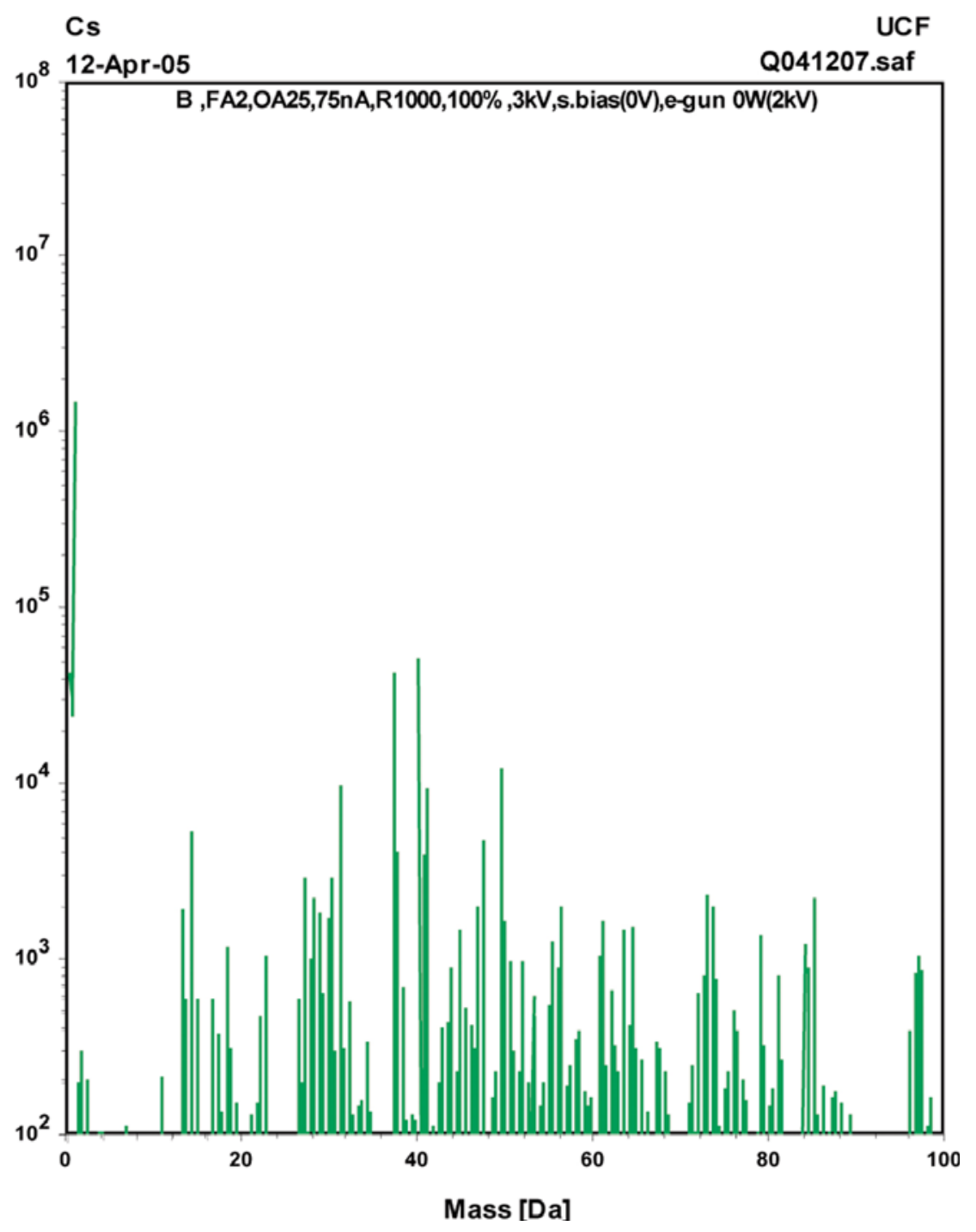

Fig. (2). SIMS of Hydrogen-Carbon-Iron After High Temperature Magnetization (17 Tesla).

heavier than iron are less after the high temperature magnetization. Moreover, it is important to note the existence of unchanged mass intensities of some nuclides with masses between 32-54 Daltons in order to demonstrate the precision of the analysis and furthermore to demonstrate the transformations of certain suitable nuclides and the intact ablation effects of some nuclides, while other nuclides remained unaltered in their natural relative abundances. The observed changes in relative amounts of some nuclides before and after are evidence of low energy nuclear reactions during the magnetization of the hot iron-graphene structures in the hydrogenous atmospheres.

The anomalous levels of ${ }^{2} \mathrm{D},{ }^{3} \mathrm{~T}$ and ${ }^{13} \mathrm{C}$ in the sample after its high temperature, magnetic annealing are a result of enhanced reverse beta and other nuclear processes of hydrogen absorbed within the iron-graphene nanocomposites. The external magnetization of the iron-graphene system causes transient ferromagnetism under the high temperature conditions with transient lattice magnetization, possibly to several thousand Tesla. The graphene under these conditions exhibits tremendous quantum Hall effects under the strong mag- netic field of the underlying iron catalyst even to the extent of chemical shifts in the covalence (diamond formation from graphite) and shifts in electron valences in hydrogen inside the Bohr radius. Such very strong magnetic fields from the iron catalyst cause a subatomic quantum Hall resistance that shifts some of the electrons to rehybridize their orbitals and even collapse their orbitals onto protons forming neutrons. Here it is suggested that subatomic quantum Hall effects under these extreme magnetic conditions inside the iron lattice cause even the resistance to orbital currents of the electrons within the $\mathrm{H}, \mathrm{C}$, and $\mathrm{Fe}$ atoms by magnetic shifts with even shift induced electric fields normal to the electronic atomic orbitals toward the nuclei for collapse of electrons onto the nuclei. Such atomic level Hall effects on lattice bonds and currents are here suggested to even explain the ferromagnetism of metals like $\mathrm{Fe}, \mathrm{Co}$ and Ni. These transient huge magnetic fields via dynamical exchange with the iron are much greater in the graphene of this composite structure relative to the recently explored magnetization of graphene (45 Tesla) by an external, laboratory electromagnet [3]. The here reported very strong dynamic magnetization of the graphene by the attached iron and the broken bonds (Fe-Fe, Fe- 
$\mathrm{C}, \mathrm{C}-\mathrm{C}, \mathrm{C}-\mathrm{H})$ under the high temperature conditions result in the greater relativistic character of the electrons and protons with even greater, tighter cyclotronic, relativistic motion and stronger subatomic quantum Hall resistance, and confinement. During these dynamic processes, slow and heavy electrons also arise in addition to the high speed relativistic electrons for reverse beta processes with surrounding protons. The huge iron magnetic field via giant magneto-resistance gives pseudo-mass to some electrons in the lattice for heavier particles on the lattice scale, but relativistic confined spinrevorbital motions of these electrons and protons on suborbital scales. The huge iron lattice magnetic fields change the dimensionality of the fermion motion in the hydrogenated graphene-iron for shift from atomic symmetry to nuclear symmetry. The resulting relativistic electrons and protons under these extreme conditions in the graphene-iron composite are able to interact via electroweak effects to form neutrons. Here it is important to consider the validity of these low energy nuclear reactions in the hot ferrocatalystgraphene system on an energetic basis. For example, huge potential energies and fields accumulate in a $6 \mathrm{~nm}$ Fe particle (about 6500 iron atoms) as it catalyzes the growth of a CNT at $750{ }^{\circ} \mathrm{C}$ at a typical rate of over $200 \mathrm{~nm} / \mathrm{s}$ under the growth conditions. Such an iron particle must handle more than 100,000 carbon atoms per sec to form the CNT at this remarkably high growth rate. During such catalysis, such an iron particle is simultaneously activating millions of $\mathrm{C}-\mathrm{C}$ bonds, which are some of the strongest possible chemical bonds. It is important to consider that the sum of the bond energies of over 100,000 such C-C bonds amounts to more than the energy to activate a nuclear reaction between $\mathrm{p}^{+}$ $\mathrm{p}^{+}$or $\mathrm{p}^{+}-\mathrm{e}^{-}$, even under the strong force and columbic $\mathrm{p}^{+}-$ $-\mathrm{p}^{+}$repulsion. The discovery here is that the huge magnetic field of the iron allows simultaneous activation and accumulation of the energy from millions of altered chemical bonds by antisymmetry and the strong exchange and correlation of the electrons and protons of these many broken bonds for the organization, accumulation and concentration of this energy of these many bodies into a few resulting relativistic particles with the magnetic confinement of the resulting relativistic particles and their consequent nuclear reactions. Such many body interactions slow some electrons and protons, whereas other electrons and protons are accelerated to tremendous speeds for novel nuclear processes within the $\mathrm{H}, \mathrm{C}$, Fe lattice. These discovered phenomena can lead to new energy technologies for ferrometal filled CNT and ferrometalgraphene composites. This discovery can also lead to new transmutation technologies for handling radioactive waste. This prospect is consistent with the recent demonstration by other investigators of the alteration of the nuclide decay lifetime by a metal lattice [12]. This discovery can also explain nuclear transformation within the interior of the earth and other planets.

\section{CONCLUSION}

On the basis of the SIMS analysis of the $\mathrm{H}, \mathrm{C}, \mathrm{Fe}$ matrix before (control) and after (sample) high temperature $\left(920^{\circ} \mathrm{C}\right)$ annealing in hydrogenous atmospheres at 17 Tesla, unusual higher levels of masses $2 \mathrm{Da}, 3 \mathrm{Da}$ and $13 \mathrm{Da}$ are observed in the sample relative to the control. On the basis of the similar $\mathrm{H}$ and $\mathrm{C}$ levels in the sample and control matrixes, these mass differences are attributed to nuclear processes under these extreme conditions. These higher levels of masses $2 \mathrm{Da}$, $3 \mathrm{Da}$, and $13 \mathrm{Da}$ are attributed to isotopes ${ }^{2} \mathrm{D},{ }^{3} \mathrm{~T},{ }^{3} \mathrm{He}$ and ${ }^{13} \mathrm{C}$ due to neutron adsorption processes of $\mathrm{H}$ and $\mathrm{C}$ in the matrix by neutron generated by reverse beta processes during the high temperature annealing under strong magnetization. These reverse beta processes during this high temperature magnetic annealing in hydrogenous atmospheres result from accumulated chemical energy stored in the broken bonds under antisymmetry during the carbon, metal and $\mathrm{H}$ bond rearrangements as $\mathrm{Fe}$ adsorb carbon, $\mathrm{CH}_{4}$ and $\mathrm{H}_{2}$ and forms graphene and some diamond. These reverse beta phenomena in Fe-graphene, Fe-CNT systems may have future technological applications as a new method of treating nuclear radioactive waste and as possible energy sources based on electroweak effects and the energy liberated during reverse beta processes. Such low energy nuclear reactions may explain the magnetism, internal heating and terrestrial nuclear reactions in the interior of the earth on the basis of such reverse beta processes in the iron media.

\section{REFERENCES}

[1] Little, R.B. U.S. 20016761871. 2001.

[2] Zhang, Y.; Tan, Y.; Stormer, H.L.; Kim. P. Nature, 2005, 438(7065), 201-204.

[3] Novoselov, K.S.; Jinag, Z.; Zhang, Y.; Morozov, S.V.; Stormer, H. L.; Zeitler, U.; Maan, J.C.; Boebinger, G.S.; Kim, P.; Geim, A.K. Science, 2007, 315(5817), 1379.

[4] Novoselov, K.S.; Geim, A.K.; Morozov, S.V.; Jiang, D.; Katsnelson, M.I.; Grigorieva, I.V.; Dubonos, S.V.; Firsov, A.A. Nature, 2005, 438, 197-200.

[5] Zhou, S.Y.; Gweon, G.H.; Graf, J.; Fedorov, A.V.; Spataru, C.D.; Diehl, R.D.; Kopelevich, Y.; Lee, D.H.; Louie, S.G.; Lanzara, A. Nat. Phys., 2006, 2(9), 595-599.

[6] Wang, X.; Chakraborty, T. Phys. Rev. B Condensed Matter Mater. Phys., 2007, 75(3), 033408/1-003408/4.

[7] Peres, N.M.R.; Castro Neto, A.H.; Guinea, F. Phys. Rev. B Condensed Matter Mater. Phys., 2006, 73(24), 241403/1-241403/4.

[8] Berger, C.; Song, Z.; Li, X.; Wu, X.; Brown, N.; Naud, C.; Mayou, D.; Li, T.; Hass, J.; Marchenkov, A.N.; Conrad, E.H.; Edward, H.; First, P.N.; de Heer, W.A. Science, 2006, 312(5777), 1191-1196.

[9] De Martino, A.; Dell'Anna, L.; Egger, R. Phys. Rev. Lett., 2007, 98(6), 066802/1-066802/4.

[10] Little. R.B. Int. J. Phys. Sci., 2006, 1(4), 175-200.

[11] Little, R.B.; Goddard, R. J. Appl. Phys., 2004, 95(5), 2702-2712.

[12] Limata, B.; Raiola, F.; Wang, B.; Yan, S.; Becker, H.W.; D'Onofrio, A.; Gialanella, L.; Roca, V.; Rolfs, C.; Romano, M.; Schuermann, D.; Strieder, F.; Terrasi, F. Eur. Phys. J. A, 2006, $28(2), 251-252$. 